\title{
Principles of Protocol to Treat Heart Blood Deficiency
}

\author{
Tong-Zheng Hong* \\ As-You-Wish Healthcare Institute, Taiwan
}

Submission: November 07, 2017; Published: December 18, 2017

*Corresponding author: Tong-Zheng Hong, As-You-Wish Healthcare Institute, Taiwan, Email: ty8876@ms24.hinet.net

\section{Introduction}

The most common and easiest access of protein and fat humans consume is meat protein [1]. It has been confirmed that protein plays a key role in the formation of atherosclerosis, a leading cause of death in the United States which is closely associated with increasing risk of heart disease [2]. Lipoprotein (a), for example, is a lipoprotein whose plasma concentration contributes to the risk of cardiovascular (CVD) disease and has been believed to be a risk factor for CVD in adults, independent of age, diet, physical activity, smoking history, ethanol consumption, and gender [3].

The coronary arteries bring blood and oxygen to the heart. Coronary artery disease (CAD) also known as atherosclerotic heart disease, coronary heart disease, or ischemic heart disease (IHD), is the most common type of heart disease, causing heart attacks.

Most of the individuals who have coronary artery disease are not aware of the disease for years as the disease progresses before the first onset of symptoms. Unfortunately, most of the symptoms and signs of coronary artery disease are noted in the advanced state of disease, often a "sudden and fatal" heart attack. Symptoms of stable ischemic heart disease include angina (chest pain) and decreased exercise tolerance. The risk of artery narrowing increases with age, smoking, high blood cholesterol, high blood pressure, and diabetes. In addition, this artery issue is more common in men and those who have close relatives with CAD.

In the pathological perspective, the disease is caused by plaque building up along the inner walls of the arteries of the heart, which narrows the arteries down and reduces blood flow to the heart. These symptoms, from the perspective of TCM, are viewed as the patterns of Qi stagnation and Blood stasis.

In TCM, the head and the upper thorax (upper Jiao) are associated with the Heart. The Heart plays a central role that any disorder affecting the Heart will also affect other organs, and vice versa. Cardiovascular disorders and organ-related specific symptoms attributed to the Heart are loss of memory, insomnia and psychosomatic problems

\section{Literature Review}

Blood (Xue) is considered to be the nourishing agent in the viewpoint of TCM, which interacts with Qi, the moving agent to maintain the circulation in body. The functions of Blood include moistening and nourishing the skin, muscles, sinews, bones and internal organs.

Blood nourishes Qi and Qi moves Blood. One is inconceivable without the other, based on the concept in TCM that Qi is "the commander of Blood, and Blood is "the mother of Qi". The circulation of Qi and Blood in the body should be constant, or the pain shall occur when the "free flow" of Qi and Blood is disrupted [4]. In other words, the relationship between Qi and Blood can be interpreted to be inter-promoting, inter-dependent, and intertransforming.

The circulation of Qi and Blood depends on the correct, interdependent functioning of Zang-Fu organs, involving in Lung, Heart, Liver, Spleen, Kidney, and San Jiao.

The Liver qi ensures Qi to circulate consistently and smoothly in the all parts of the body, connecting to the Spleen and promoting the digestion functions to produce and hold Blood. Mechanisms in the imbalance of Qi and Blood include external factors like Wind and Heat, emotional disturbance, bad diet and physic trauma, and improper exertion in activities, such as sex or excessive study and thinking [4].

Pain is mainly a present of disturbances in the circulation of Qi and Blood, which indicates the stagnation of Qi and stasis of Blood, or Qi deficiency and Blood deficiency. The organ that causes of these is Heart because Heart dominates Blood and blood vessels, and is the root controller of Zang-Fu organs [5].

Angina is the common symptom in patients with coronary disease. There could be various causative factors leading to pain, but the main pathology is either due to blockage and obstruction, or deficiency of Blood. Malfunction and dysfunction 
of rotting and ripening of Stomach, which controls digestion, and transforming and transporting of Spleen, which produces and holds Blood, occur when these two organs are invaded by the stagnated Liver qi.

Blood deficiency results actually from hypo function in Blood production, excessive Blood consumption of Essence and Blood with prolonged illness or over-exhaustion. The palpitations occur as long as Blood supply to the Heart is insufficient.

\section{Principles of Protocol}

An experienced TCM practitioner can only rely on four skills for diagnosis to identify Patterns and prescriptions, compared to Western medicine physicians. In other words, Patterns, which distinguish TCM from conventional medicine, should be the key concern rather than diseases for TCM and acupuncture practitioners in treatment.

Blood becomes static when Qi is impeded. For better results, Blood mobilization and Qi regulation need treatment at the same time. On the other hand, it deserves attention that deficiency of Blood may be seen as the source of dampness when it is closely linked to Spleen qi deficiency.

The most critical notion that "Feng (Wind), the beginner of the illness" is presented in Huang Di Nei Jing (Essential Questions of Yellow Emperor's Inner Classic). This notion attracts much attention because Wind is considered the major cause of illness with its pernicious influence. It is also stated Blood Xu (deficiency of Blood) generates Wind, which may refer to pain, spasm and hypotension in the patient with Heart Blood deficiency.

In SùWèn (Essential Questions) of Huang Di Nei Jing, the notion "choose Shu-stream to treat Zang; He-sea to treat Fu" is the best guideline for prescriptions.

For the purpose of balance of Qi and Blood, LV 3 (Tai chong) and ST 36 (Zusanli) are essential acupoints in the protocol on the basis of Qi-Blood balance, shown in Figure 1. Clinical experiences show it is beyond doubt that these two acupoints essential in the protocol function well to the author's expectations.

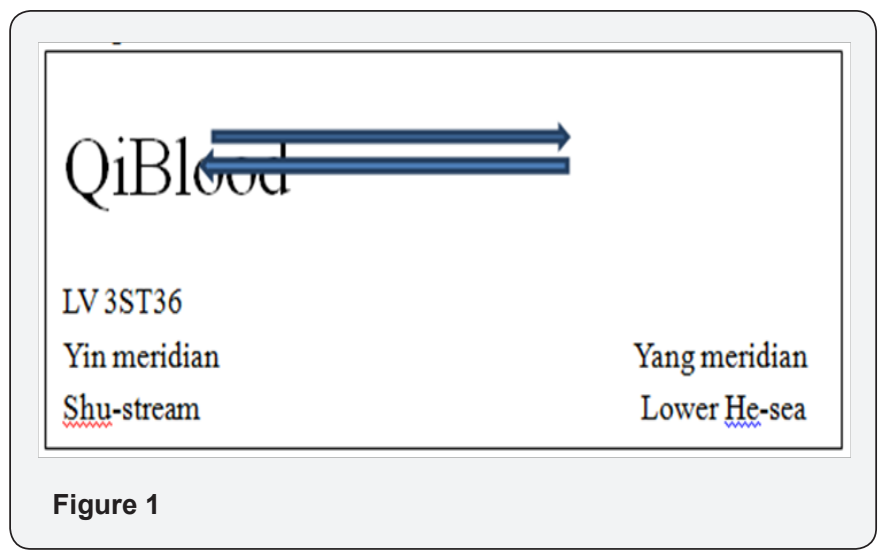

For Heart Blood deficiency caused by LV Qi stagnation and Blood stasis, the Shu-stream point of Heart meridian HT 7 (Shenmen) is added to nourish Heart Blood.

\section{Conclusion}

Acupuncture tends to produce good results in the treatment of functional heat problems as well as difficulty caused by disorders of the autonomic nervous system.

The beginning stages of coronary heart disease without any advanced narrowing coronary arteries are well suited for acupuncture interventions. However, good results cannot be expected if key symptoms are not identified.

\section{References}

1. Clifton PM (2011) Protein and coronary heart disease: the role of different protein sources. Curr Atheroscler Rep 13(6): 493-498.

2. Clinton Colmenares. Protein links heart disease, diabetes.

3. Schreiner PJ, Morrisett JD, Sharrett AR, Patsch W, Tyroler HA, et al. (1993) Lipoprotein(a) as a risk factor for preclinical atherosclerosis. Arterioscler Thromb 13(6): 826-833.

4. Sun P (2011) Treatment of pain with chinese herbs and acupuncture. Churchill Livingstone, New York, USA.

5. Zhu B, Wang H (2010) Basic theories of traditional chinese medicine. People's Military Medical Press, Pennsylvania, USA. 